\title{
Real-time assessment of cardiac perfusion, coronary angiography, and acute intravascular thrombi using dual-channel near-infrared fluorescence imaging
}

\author{
Eiichi Tanaka, MD, PhD, ${ }^{\text {a,b }}$ Frederick Y. Chen, MD, PhD,${ }^{c}$ Robert Flaumenhaft, MD, PhD, ${ }^{d}$ \\ Gwenda J. Graham, PhD, ${ }^{\mathrm{d}}$ Rita G. Laurence, BS, ${ }^{\mathrm{a}}$ and John V. Frangioni, MD, $\mathrm{PhD}^{\mathrm{a}, \mathrm{e}}$
}

\begin{abstract}
Objectives: We have developed an image-guided surgical system based on invisible near-infrared fluorescent light. Presently, the only clinically available near-infrared fluorophore is indocyanine green, which fluoresces at approximately $800 \mathrm{~nm}$ and is used for coronary angiography. Our objective was to determine whether methylene blue, already US Food and Drug Administration approved for other indications, has useful near-infrared fluorescence properties for image-guided cardiac surgery.
\end{abstract}

\begin{abstract}
Methods: The optical properties of methylene blue were measured after dissolution in $100 \%$ serum. Biodistribution and clearance were quantified in organs and tissue after intravenous bolus injection of $2 \mathrm{mg} / \mathrm{kg}$ methylene blue in 3 rats. Coronary arteriography and cardiac perfusion were imaged in real time after intravenous bolus injection of $1 \mathrm{mg} / \mathrm{kg}$ methylene blue in 5 pigs with coronary obstructions. Coronary angiography and acute thrombi were assessed by using 800-nm fluorophores, indocyanine green, and IR-786-labeled platelets, respectively.
\end{abstract}

Results: The peak absorbance and emission of methylene blue as a near-infrared fluorophore occur at $667 \mathrm{~nm}$ and $686 \mathrm{~nm}$, respectively. After intravenous injection, methylene blue provides highly sensitive coronary angiography. A lipophilic cation, methylene blue is extracted rapidly into tissue, with myocardium displaying unusually high uptake. Methylene blue permits real-time visualization and quantitative assessment of myocardial perfusion. Because of absent spectral overlap, use of 2 independent fluorophores in our imaging system permits simultaneous quantification of perfusion, venous drainage, and/or intravascular thrombi.

Conclusions: Methylene blue is an effective near-infrared fluorophore that provides direct visualization of coronary arteriography and cardiac perfusion. In conjunction with approximately $800-\mathrm{nm}$ near-infrared fluorophores, important functional assessments during cardiac surgery are also possible.

Immediate and long-term outcomes of coronary artery bypass grafting $(\mathrm{CABG})$ are typically dependent on the quality of the anastomosis, the conduit, and the distal myocardial runoff. In current practice there is typically no standardized approach for the intraoperative assessment of the anastomosis or the distal runoff. Although postoperative aspirin administration and increased use of arterial grafting have improved early and late graft patency, perioperative graft occlusions are still reported at a rate of between $4 \%$ and $12 \%{ }^{1,2}$ If such graft failures can be recognized intraopera-

\footnotetext{
From the Division of Hematology/Oncology, ${ }^{\text {a }}$ the Division of Hemostasis and Thrombosis, ${ }^{\mathrm{d}}$ and the Department of Radiology, ${ }^{\mathrm{e}}$ Beth Israel Deaconess Medical Center, Boston, Mass; the Department of Surgical Oncology, ${ }^{\mathrm{b}}$ Hokkaido University Graduate School of Medicine, Sapporo, Japan; and the Division of Cardiac Surgery, ${ }^{c}$ Brigham and Women's Hospital, Boston, Mass.

Supported by National Institutes of Health grants R01-CA-115296 (to J.V.F.), R01EB-005805 (to J.V.F.), and R01-HL-063250 (to R.F.) and an Established Investigator Award from the American Heart Association (to R.F.).

Received for publication April 11, 2008; revisions received July 29, 2008; accepted for publication Sept 6, 2008.

Address for reprint: John V. Frangioni, MD, PhD, Beth Israel Deaconess Medical Center, Room SL-B05, 330 Brookline Ave, Boston, MA 02215 (E-mail: jfrangio@bidmc.harvard.edu).

J Thorac Cardiovasc Surg 2009;138:133-40

0022-5223/\$36.00

Copyright (c) 2009 by The American Association for Thoracic Surgery

doi:10.1016/j.jtcvs.2008.09.082
}

tively, they might be correctable. Because graft patency and completeness of revascularization are 2 important prognostic factors for long-term survival after coronary surgery, a reliable and well-validated method to assess anastomoses and perfusion would be helpful.

Near-infrared (NIR) fluorescent light (700-900 nm), which is invisible to the human eye, provides an extremely high signal/background ratio without changing the look of the tissue being imaged. ${ }^{3}$ Our laboratory has previously developed a 1-channel intraoperative NIR fluorescence imaging system capable of visualizing otherwise invisible relevant surgical anatomy by using a color video camera and NIR fluorescence from an NIR camera. ${ }^{4}$ Using a variety of large-animal model systems, we have shown that NIR fluorescence can be used for effective image-guided sentinel lymph node mapping,${ }^{5}$ cardioplegia delivery, ${ }^{6}$ ureter identification, ${ }^{7}$ and extrahepatic bile duct identification. ${ }^{8}$ Our group has also demonstrated that autologous washed platelets can be rendered NIR fluorescent at $800 \mathrm{~nm}$ and, when injected intravenously, will home to sites of intravascular thrombi to provide high-sensitivity detection. ${ }^{9}$

There are presently 2 fundamental limitations to the widespread clinical use of NIR fluorescence technology: the availability of US Food and Drug Administration (FDA)- 


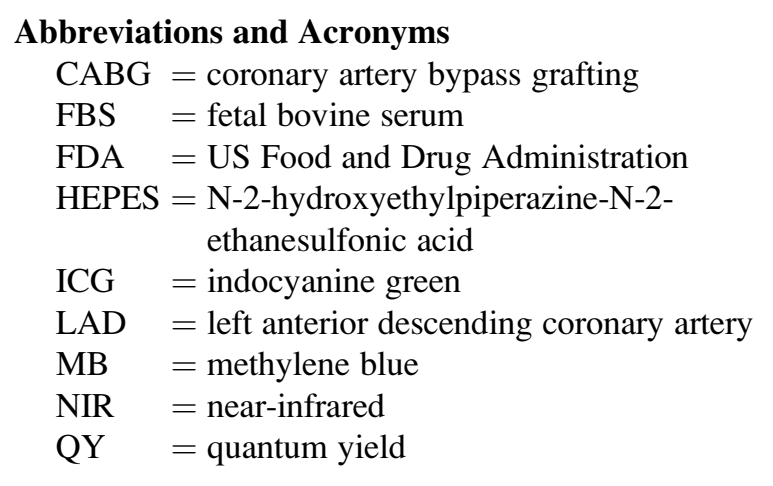

approved NIR fluorophores and the availability of imaging systems capable of visualizing more than 1 NIR fluorophore simultaneously. Indocyanine green (ICG) is a small-molecule blood pool agent that fluoresces at $800 \mathrm{~nm}$ and has previously been used for coronary angiography during CABG surgery. ${ }^{10-12}$ Methylene blue (MB) is another FDA-approved fluorophore that heretofore has not been used for intraoperative imaging. In this study we hypothesized that MB has independent and nonoverlapping optical and pharmacokinetic properties compared with ICG and that it can therefore be imaged concomitantly with ICG and other 800-nm fluorophores to provide simultaneous and unique image guidance during cardiac surgery.

\section{MATERIALS AND METHODS}

\section{Dual-Channel NIR Fluorescence Imaging System}

Our FLARE (Fluorescence-Assisted Resection and Exploration) largeanimal, single-channel intraoperative NIR fluorescence imaging system has been described in detail previously. ${ }^{4,13}$ We recently developed a dualNIR version that includes a light-emitting diode-based light source that generates $1 \mathrm{~mW} / \mathrm{cm}^{2}$ of 400 - to $650-\mathrm{nm}$ " white" light, $2.5 \mathrm{~mW} / \mathrm{cm}^{2}$ of $670-\mathrm{nm}$ excitation light for NIR fluorescence channel 1 , and $5 \mathrm{~mW} / \mathrm{cm}^{2}$ of $760-\mathrm{nm}$ NIR excitation light for NIR fluorescence channel 2. Photon collection was achieved with footswitch-controlled zoom optics that maintain separation of the white light and 2 NIR fluorescence emission (700 and $800 \mathrm{~nm}$, respectively) channels. The system has a spatial resolution of $625 \mu \mathrm{m}$ at a field of view of $20 \times 15 \mathrm{~cm}$ and $125 \mu \mathrm{m}$ at a field of view of $4 \times 3 \mathrm{~cm}$. Color video images were acquired on an IMI tech IMC-80F camera (IMI Tech, Seoul, Korea). NIR fluorescence images were acquired on two 12-bit Orca-AG cameras (Hamamatsu, Bridgewater, NJ) used within their linear range. After computer-controlled camera acquisition, standard (white light) and 2 functional (NIR fluorescent light) video images can be displayed separately and merged. The imaging system was suspended over the surgical field with an articulating arm, with a working distance of 18 inches above the subject. Data were acquired and quantified on a Dell computer by using custom LabVIEW 7.1 software (National Instruments, Austin, Tex).

\section{NIR Fluorophore Preparation}

Ten milligrams per milliliter (1\%) sterile vials of MB in water and 25-mg sterile vials of ICG (aka CardioGreen) powder were purchased from Akorn, Inc (Decatur, Ill). The perchlorate salt of IR-786 (CAS \#102185-03-5) was purchased from Sigma-Aldrich (St Louis, Mo), and bioactive, autologous NIR fluorescent pig platelets were prepared as described previously. ${ }^{9}$ Briefly, platelet-rich plasma was isolated by means of centrifugation of $200 \mathrm{~mL}$ of whole blood at $200 \mathrm{~g}$ for 20 minutes. Platelets were isolated from plateletrich plasma by means of centrifugation at $1400 \mathrm{~g}$ for 10 minutes in the presence of $50 \mathrm{ng} / \mathrm{mL}$ prostaglandin $\mathrm{E}_{1}$ and $10 \%$ ( $\left.\mathrm{vol} / \mathrm{vol}\right)$ acid citrate/dextrose, $\mathrm{pH} 4.6$, and resuspended at a concentration of $4 \times 10^{8}$ platelets $/ \mathrm{mL}$ in Tyrode's-N2-hydroxyethylpiperazine-N-2-ethanesulfonic acid (HEPES) buffer. The washed platelets $\left(3.6 \times 10^{10}\right.$ total $)$ in Tyrode's-HEPES buffer were labeled with $2 \mu \mathrm{mol} / \mathrm{L}$ IR-786 for 30 minutes at room temperature, as described previously. ${ }^{9}$

\section{Measurement of MB's Optical Properties}

All measurements were performed in $100 \%$ fetal bovine serum (FBS). Absorbance and fluorescence were measured with fiberoptic HR2000 absorbance $(200-1100 \mathrm{~nm})$ and USB2000FL fluorescence $(350-1000 \mathrm{~nm}$ ) spectrometers (Ocean Optics, Dunedin, Fla). NIR excitation was provided by a $5-\mathrm{mW}, 654-\mathrm{nm}$ laser diode. Fluorescence quantum yield (QY) of MB in FBS was calculated by using oxazine 725 in ethylene glycol $(\mathrm{QY}=19 \%)^{14}$ as a calibration standard under conditions of matched absorbance (absorbance $=0.08$ ) at $654 \mathrm{~nm}$.

\section{Surgical Preparation}

All animals were used under the supervision of an approved institutional protocol. Three hundred-gram Sprague-Dawley rats from Charles River Laboratories (Wilmington, Mass) were anesthetized with $65 \mathrm{mg} / \mathrm{kg}$ intraperitoneal pentobarbital and were ventilated by means of tracheostomy with an MRI-1 ventilator (CWE, Inc, Ardmore, Pa). Bilateral thoracotomy and laparotomy were performed, and the lungs, heart, liver, kidney, inferior vena cava, and skeletal muscles at the abdominal wall were exposed.

Adult female Yorkshire pigs (mean weight, $30 \mathrm{~kg}$ ) were purchased from E.M. Parsons and Sons (Hadley, Mass). General anesthesia was induced with $4.4 \mathrm{mg} / \mathrm{kg}$ intramuscular Telazol (Fort Dodge Labs, Fort Dodge, Iowa). Once sedated, animals were intubated with a cuffed endotracheal tube, and anesthesia was maintained with $2 \%$ isoflurane/balance $\mathrm{O}_{2}$. Continuous oxygen saturation, body temperature in the oral cavity, and 3-lead electrocardiographic results were monitored throughout the experiment. After a median sternotomy was performed, the heart was suspended in a pericardial cradle. Animals received intravenous lidocaine $(50 \mathrm{mg})$ through a left external jugular vein catheter before clamping a diagonal branch of the left anterior descending coronary artery (LAD) to prevent ventricular fibrillation.

\section{MB Biodistribution and Clearance}

Two milligrams per kilogram of MB was injected intravenously as a bolus over 30 seconds. Under conditions of constant fluence rate and field of view, 700-nm NIR fluorescence images of the lung, heart, liver, kidney, inferior vena cava, and skeletal muscle at the abdominal wall were acquired every $500 \mathrm{~ms}$ until 1 minute and then at 2 minutes, 5 minutes, and every 5 minutes thereafter for a total of 60 minutes.

\section{Coronary Arteriography and Myocardial Perfusion Imaging With MB}

One milligram per kilogram of MB was injected intravenously as a bolus over 30 seconds. Images were acquired every $500 \mathrm{~ms}$ until 1 minute and then at 2 minutes, 5 minutes, and every 5 minutes thereafter for a total of 60 minutes. For some experiments, a diagonal branch of the LAD was clamped before $\mathrm{MB}$ injection. Fifteen minutes after interrupting arterial flow, the clamp was removed, and ICG $(0.06 \mathrm{mg} / \mathrm{kg})$ was injected intravenously. Fifteen minutes after ICG injection, the animal was killed, the heart was removed, and the cut surface was imaged.

\section{Induction of Intravascular Thrombi in Coronary Arteries}

Up to 2 hours before thrombus induction, $3.6 \times 10^{10}$ NIR fluorescent platelets were infused intravenously. A $1 \mathrm{~cm} \times 1 \mathrm{~cm}$ piece of filter paper was dipped in $50 \%$ (wt/wt) aqueous $\mathrm{FeCl}_{3}$ and then placed over a diagonal 


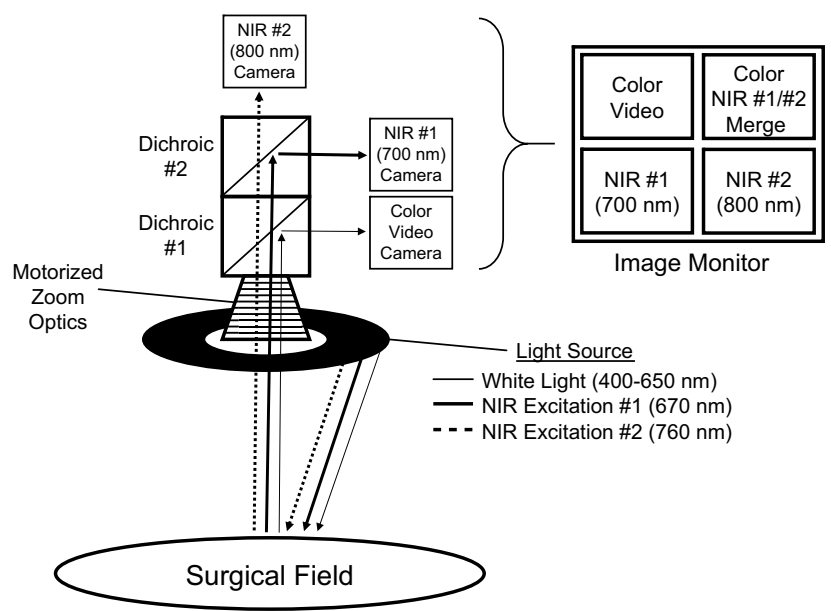

FIGURE 1. Image-guided surgical system equipped with 2 near-infrared (NIR) fluorescence channels: schematic of the imaging system showing light paths for white light, NIR channel 1 excitation $(670 \mathrm{~nm})$ and emission $(700 \mathrm{~nm})$, and NIR channel 2 excitation $(760 \mathrm{~nm})$ and emission $(800 \mathrm{~nm})$. All images were acquired and displayed simultaneously and in real time.

branch of the LAD to induce thrombi. Thrombi could be detected with 800 nm NIR fluorescence within 30 to 45 minutes of $\mathrm{FeCl}_{3}$ application, after which time $1 \mathrm{mg} / \mathrm{kg}$ MB was injected intravenously, as described above.

\section{Quantitation and Statistical Analysis}

NIR fluorescence was quantified by using a constant-sized region of interest over reproducible areas of the heart and coronary arteries: midanterior right ventricular free wall, midanterior left ventricular free wall, bifurcation<smiles>CN(C)c1ccc2nc3ccc(=[N+](C)C)cc-3sc2c1</smiles>

A

M.W. 321

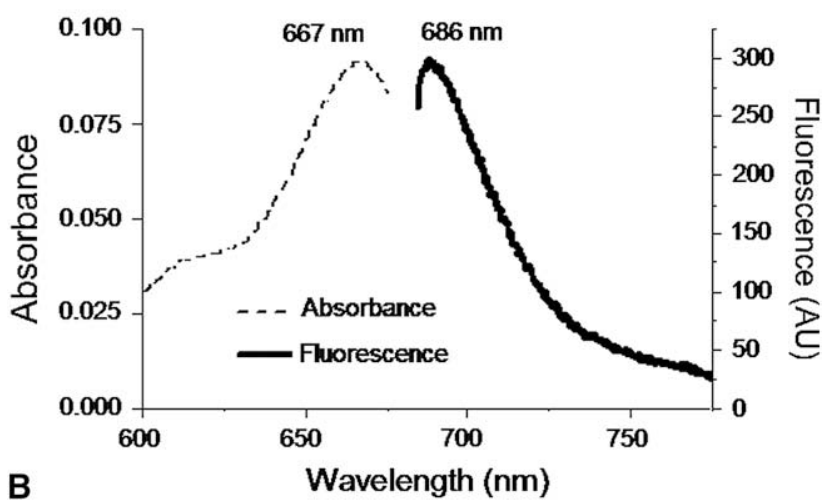

FIGURE 2. Chemical and optical properties of methylene blue. A, Chemical structure and molecular weight (M.W.; in daltons) of methylthioninium chloride (methylene blue). B, Absorbance (left axis) and fluorescence (right axis; 654-nm excitation) for $2 \mu \mathrm{mol} / \mathrm{L}$ methylene blue in $100 \%$ FBS.
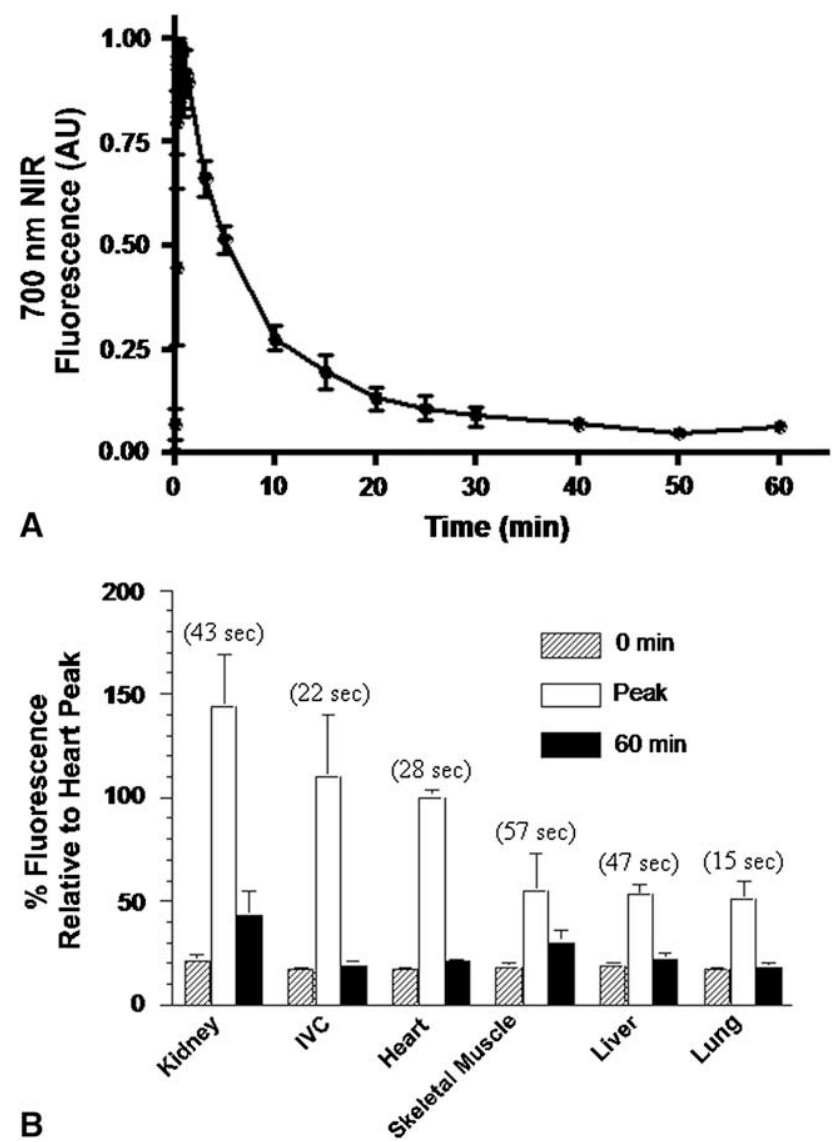

FIGURE 3. In vivo biodistribution and clearance of methylene blue. A, Kinetics of 700-nm MB near-infrared (NIR) fluorescence in rat myocardium. Shown are means \pm standard errors of the mean signal intensity from 3 independent animals. B, Ratio of NIR fluorescence intensity of the myocardium relative to various vital organs and tissues after intravenous injection of $2 \mathrm{mg} / \mathrm{kg} \mathrm{MB}$ in rats $(\mathrm{n}=3)$. Shown are values at 0 minutes, at the time (parentheses) of peak fluorescence in the organ/tissue under study, and at 60 minutes. $I V C$, Inferior vena cava.

of the second diagonal branch, and pericardium. A Mann-Whitney $U$ test was used for statistical comparison of parameters.

\section{RESULTS}

\section{Real-Time, Dual-Channel Intraoperative NIR} Fluorescence Imaging System

A schematic of the imaging system used in this study is shown in Figure 1. Its 18-inch working distance and articulated arm ensured unobtrusive imaging during cardiac surgery, and its articulated arm permitted precise positioning. By suppressing motion artifacts, cardiac gating technology preserved high-resolution imaging of the beating heart (see below). The color video, 700-nm (NIR fluorescence channel 1), and 800-nm (NIR fluorescence channel 2) images were acquired simultaneously to display anatomy and function together, and NIR fluorescence channels were pseudocolored and overlaid on top of the color video image to create a separate merged image. All images were refreshed at rates up to 

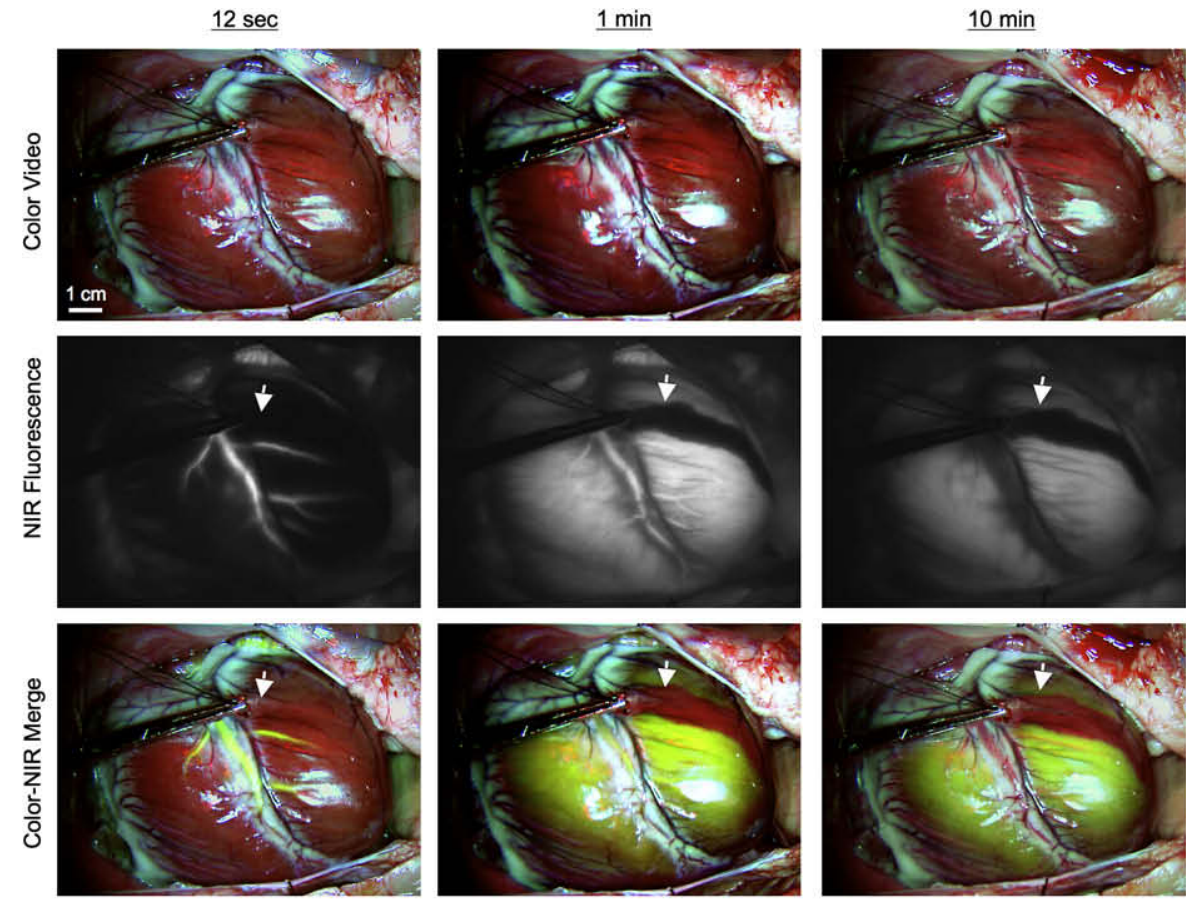

A
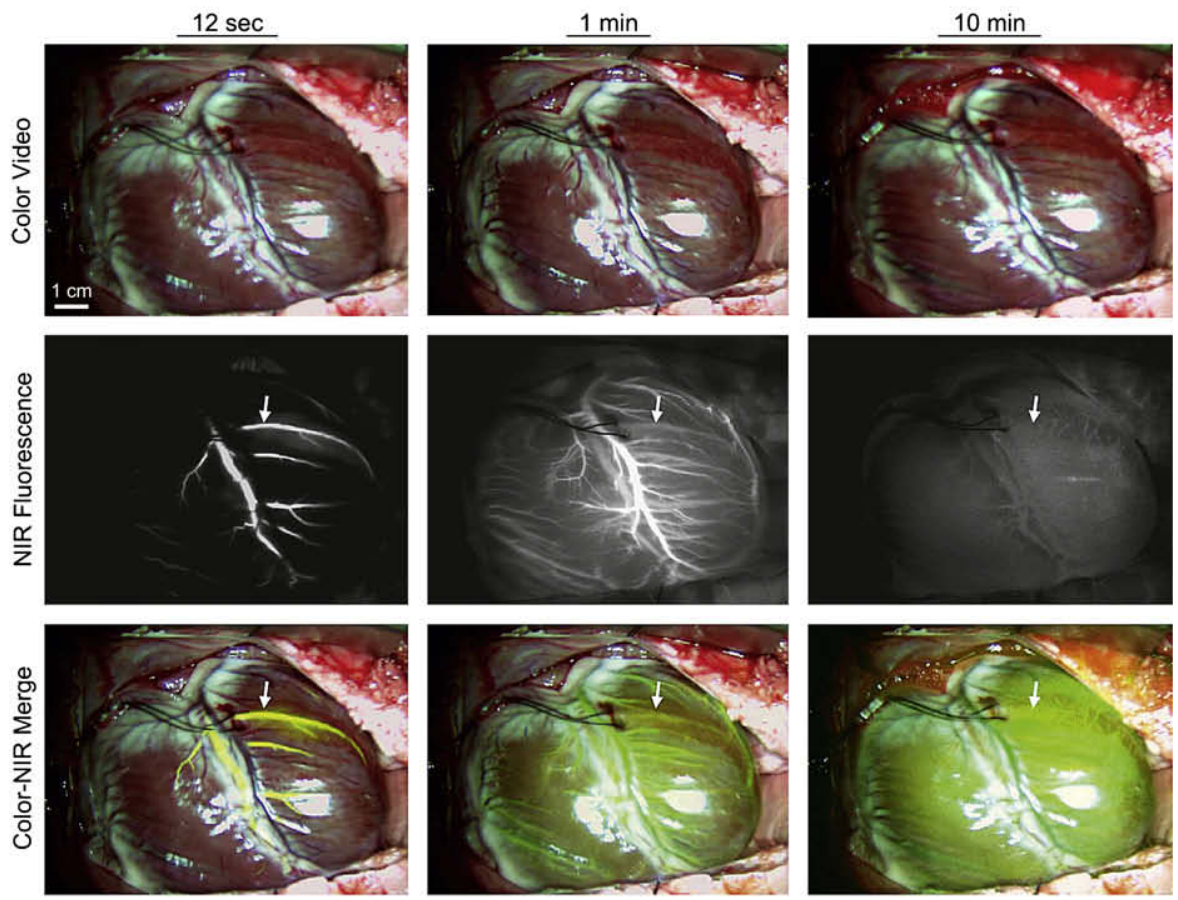

B

FIGURE 4. Real-time simultaneous assessment of myocardial perfusion and coronary angiography using methylene blue $(M B)$ and indocyanine green (ICG). A, The arterial phase (12 seconds), venous phase (1 minute), and late phase (10 minutes) after intravenous bolus injection of $1 \mathrm{mg} / \mathrm{kg} \mathrm{MB}$ into pigs are shown. A diagonal branch of the left anterior descending coronary artery $(L A D)$ was clamped immediately before MB injection, with the perfusion defect easily detected (arrows). Shown are color video (top row), near-infrared (NIR) fluorescence (middle row), and a pseudocolored (lime green) merge of the two (bottom row). NIR fluorescence images were acquired with a 100-ms exposure time and are displayed with identical normalizations. Images are representative of 5 independent animals. B, The arterial phase ( 12 seconds), venous phase ( 1 minute), and late phase (10 minutes) after declamping of the diagonal branch and intravenous bolus injection of $0.06 \mathrm{mg} / \mathrm{kg}$ ICG into the pig from panel A are shown. Arrows mark the location of the previous perfusion defect seen with MB. Shown are color video (top row), NIR fluorescence (middle row), and a pseudocolored (lime green) merge of the two (bottom row). NIR fluorescence images were acquired with a 100-ms exposure time and are displayed with identical normalizations. Images are representative of 5 independent animals. 


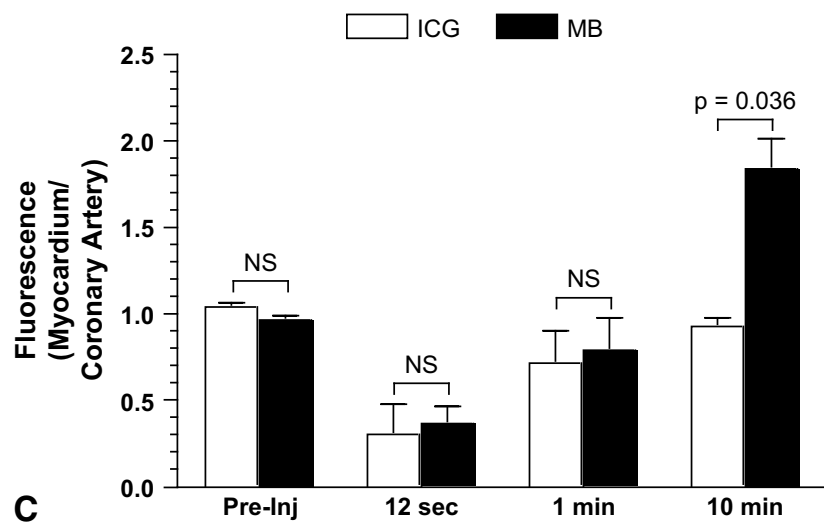

FIGURE 4. (Continued). C, Quantitative analysis of the ratio of myocardial NIR fluorescence to coronary artery NIR fluorescence 10 minutes after intravenous bolus injection of either ICG or MB is shown. Shown are the means \pm standard errors of the mean of 5 independent animals, along with the statistical comparison of the 2 NIR fluorophores at each time point.

15 times per second. Because NIR excitation and emission light are invisible to the human eye, the surgical field appears completely normal.

\section{Chemical and Optical Properties of MB}

MB (methylthioninium chloride) is a lipophilic, cationic (charge $=+1)$, 321-d small molecule with a distributed electron resonance (Figure 2, $A$ ). In 100\% serum, peak absorbance and emission were found to be $667 \mathrm{~nm}$ and $686 \mathrm{~nm}$, respectively (Figure 2, B). Its extinction coefficient at 667 $\mathrm{nm}(\mathrm{e} 667 \mathrm{~nm})$ was found to be $46,000 \mathrm{M}^{-1} \mathrm{~cm}^{-1}$. QY in $100 \%$ serum was $2.5 \%$. The product of e667nm and QY indicated low-to-moderate total fluorescence compared with that seen with other NIR fluorophores, such as ICG (ePeak absorbance $=166,000 \mathrm{M}^{-1} \mathrm{~cm}^{-1}$ and QY $=9 \%$ in serum $){ }^{5}$

\section{In Vivo Biodistribution and Clearance}

MB displays no protein binding after intravenous injection. ${ }^{15}$ After intravenous bolus injection of $2 \mathrm{mg} / \mathrm{kg}$ into a rat, region-of-interest analysis over the myocardium revealed high uptake of $\mathrm{MB}$, peaking at 28 seconds, and slow washout, with the NIR fluorescence signal returning to background levels by 40 to 60 minutes (Figure 3, A). Other vital organs and tissues also exhibited uptake of $\mathrm{MB}$, with peak fluorescence occurring between 15 and 57 seconds after injection (Figure 3, B). The NIR fluorescence signal from all except the kidney returned to baseline by 60 minutes. Excretion of $\mathrm{MB}$ was found to be both renal and hepatic (data not shown).

\section{Coronary Arteriography and Myocardial Perfusion Imaging With MB}

In a human-sized pig, 700-nm MB fluorescence provided sensitive coronary arteriography, with the arterial phase peaking 12 seconds after intravenous bolus injection (Figure 4, A). Unlike blood pool agents, such as ICG, and like other lipophilic cations, ${ }^{16} \mathrm{MB}$ was extracted completely by the myocardium. This eliminates the venous phase but also provides direct visualization of the myocardial perfusion or arterial runoff (Figure $4, A$ ). As seen in the rat, peak NIR fluorescence in the myocardium occurred at 1 minute after $\mathrm{MB}$ injection, persisted for more than $10 \mathrm{~min}$ utes (Figure $4, A$ ), and returned to baseline value by 40 to 60 minutes. Importantly, MB NIR fluorescence contrast was so high that myocardium with an induced perfusion defect could be detected with high sensitivity (Figure 4, A).

The perfusion defect was reversed, and $0.06 \mathrm{mg} / \mathrm{kg}$ ICG was injected intravenously as a bolus. By using the dualchannel capability of the imaging system, the opened artery could be assessed in real time, with peak fluorescence occurring at 12 seconds after injection (Figure $4, B$ ). Because ICG uptake into the myocardium is negligible, venous filling can be assessed (Figure 4,B) but not perfusion. Quantitative comparison of the ratio between myocardial fluorescence and coronary artery fluorescence at various time points after intravenous injection of ICG and MB is shown in Figure 4, $C$, and confirms the qualitative results. Taken together, $\mathrm{MB}$ and ICG, in conjunction with the dual-channel imaging system, permitted simultaneous, real-time, quantitative assessment of arterial filling, venous filling, and myocardial perfusion during cardiac surgery in the beating heart.

\section{Simultaneous Detection of Coronary Arterial Flow, Myocardial Perfusion, and Acute Thrombi}

Presently, there is no method available for the sensitive detection of intravascular thrombi during cardiac surgery. We have previously demonstrated that intravenously injected 800-nm NIR fluorescent platelets will home to the sites of intravascular thrombi to provide high-sensitivity detection. ${ }^{9}$ The present study demonstrates that the combination of 800-nm NIR fluorescent platelets and 700-nm MB, in conjunction with the dual-channel imaging system, can be used to detect thrombi forming in even small branches of the coronary arteries and, importantly, can provide functional information about vessel patency. The diagonal branch of the LAD harboring a thrombus is completely occluded, as assessed with MB (Figure 5). Indeed, the thrombus shown in Figure 5 is the smallest seen in 4 animals, measuring submillimeters in size, which highlights the sensitivity of the technology and the motion-suppressing, highresolution capabilities of the imaging system.

\section{DISCUSSION}

In CABG there is still no widely accepted or broadly used intraoperative technique to assess the quality of the anastomosis or the bypass graft itself. The clinical consequences of early graft failure are devastating. In a recent report the 30-day mortality among patients with early postoperative graft failure was more than $9 \% .^{17}$ Because graft patency is the predominant factor in long-term survival and avoidance of reintervention after $\mathrm{CABG}$ surgery, an intraoperative 

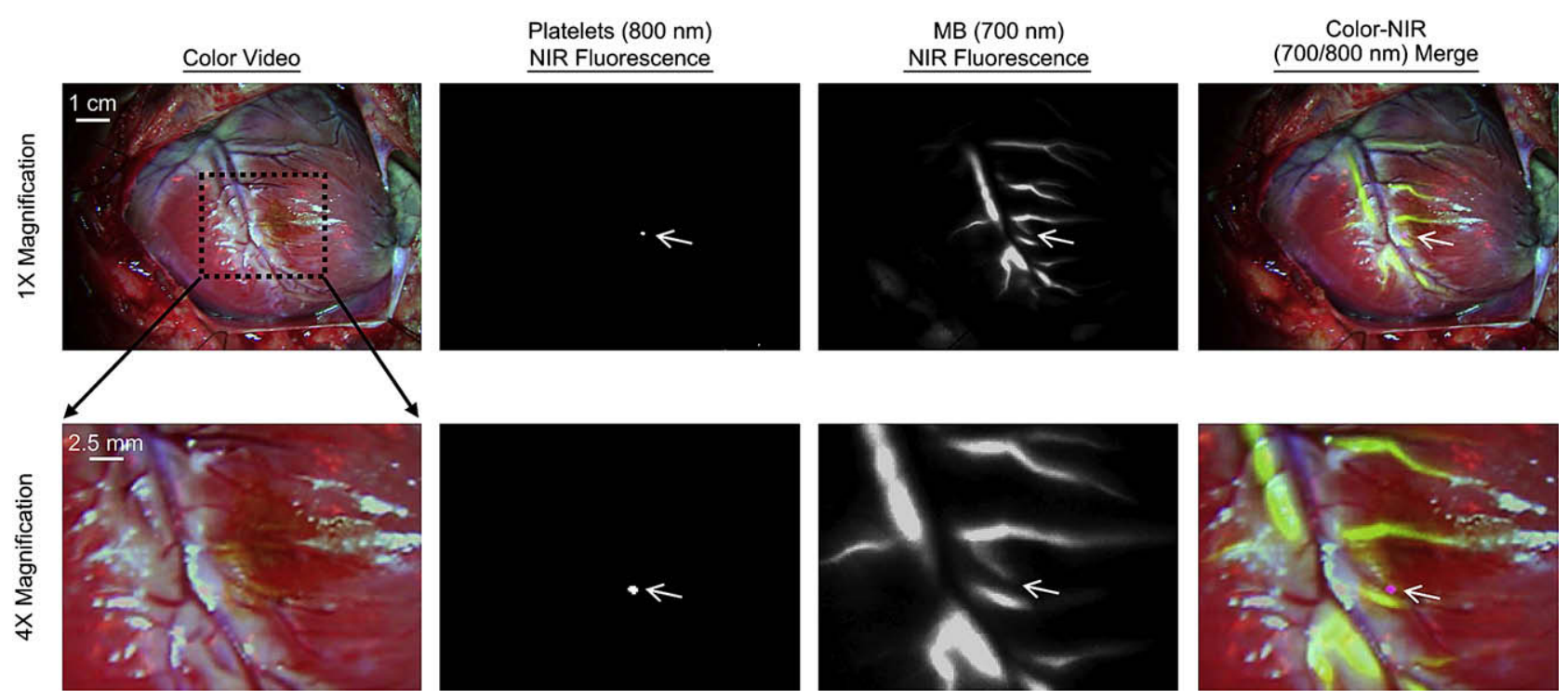

FIGURE 5. Real-time simultaneous assessment of acute coronary artery thrombi and coronary artery patency. A coronary artery thrombus was induced in a diagonal branch of the left anterior descending coronary artery $(L A D)$ by means of treatment with $\mathrm{FeCl}_{3}$ (center of dotted rectangle). Thrombus size and location were quantified by means of intravenous injection of IR-786-labeled autologous platelets (800-nm fluorescence; pseudocolored in magenta in the merged image). Coronary artery patency (and tissue perfusion) was quantified by means of intravenous bolus injection of $1 \mathrm{mg} / \mathrm{kg} \mathrm{MB} \mathrm{(700-nm} \mathrm{fluorescence;}$ pseudocolored in lime green in merged image). Shown are color video (first column), 800-nm NIR fluorescence (second column), 700-nm NIR fluorescence (third column), and a pseudocolored merge of the three (fourth column) at 12 seconds after MB injection. The area within the dotted rectangle in the top row is shown magnified 4-fold in the bottom row. NIR fluorescence images were acquired with a 100-ms exposure time and are displayed with identical normalizations. Images are representative of 4 independent animals.

means of assessing revascularization would be extremely helpful. This need to assess intraoperative patency and myocardial perfusion has been emphasized in recent studies that demonstrate significant surgeon-dependent variations in graft patency in off-pump CABG procedures. ${ }^{18}$

Among graft assessment techniques currently available, iodine-based coronary angiography remains the gold standard for postoperative assessment. However, catheterization and fluoroscopy equipment is typically not available in the operating room. Although the assessment of graft patency by using thermal angiography, ${ }^{19}$ an electromagnetic flowmeter, ${ }^{20}$ or Doppler flow measurement, ${ }^{21}$ have been attempted clinically, such techniques generally do not provide images and have not been routinely implemented in the field of surgery thus far. ${ }^{22}$ Transit-time ultrasonic flow measurement has also become a popular method to assess graft patency. ${ }^{23,24}$ Although transit-time ultrasonic flow measurement is rapid and simple to use, the technique does not produce an image, and interpretation of flow data is often difficult and less intuitive than an angiographic presentation of the graft status.

ICG angiography is a promising new technique for intraoperative assessment during CABG surgery. ${ }^{11,12}$ Recent publications demonstrate that ICG angiography might be an effective and reproducible method for the evaluation of graft status. ${ }^{10}$ In this study we describe a dual-channel NIR fluorescence imaging system capable of visualizing 800-nm ICG fluorescence simultaneously with 700-nm
MB fluorescence. We also demonstrate that MB, already FDA-approved for intravenous use in the treatment of methemoglobinemia at a dose of 1 to $2 \mathrm{mg} / \mathrm{kg}$, has unusually high uptake into the myocardium and thus serves as a simple, yet powerful visual tracer of myocardial perfusion and arterial runoff. ICG is a true blood pool agent; that is, it flows into arteries and then out of veins, providing approximately 3 to 4 seconds of imaging in each structure as it passes through. Although ICG can be used as a surrogate for cardiac perfusion, ${ }^{25}$ quantitation is difficult, and perfusion is never actually visualized. MB, on the other hand, provides imaging of arteries but is then efficiently extracted into the myocardium during its first pass through the capillaries. Importantly, because there is no spectral overlap between ICG and $\mathrm{MB}$, our dual-channel imaging system permits the complementary information from both to be imaged simultaneously.

However, MB does have limitations for clinical use. A factitious and transient decrease in pulse oximetry readings is always observed after its intravenous injection. ${ }^{26} \mathrm{MB}$ 's extinction coefficient and QY in serum are relatively low, and serious adverse reactions have been reported when used at doses of $7.5 \mathrm{mg} / \mathrm{kg} .{ }^{27}$ Even at $1 \mathrm{mg} / \mathrm{kg}$, it is usually administered as a slow bolus over 15 minutes rather than the 15 -second administration used in this study. Although no acute toxicity was seen in either rats or pigs, this is an important consideration for translation to patients. Of course, absolute fluorescence intensity is the product of the $670-\mathrm{nm}$ 
NIR excitation fluence rate, MB concentration in the myocardium, and camera exposure time. In experiments not shown, the dose could be reduced 2-fold when either the fluence rate or exposure time was doubled and 4-fold when both were doubled. Because there is a tradeoff between exposure time and resolution as a result of cardiac motion, we expect that the fluence rate will be the key to decreasing the MB dose, with a maximum likely being $10 \mathrm{~mW} / \mathrm{cm}^{2}$ (4-fold higher than used in this study, leading to a 4-fold lower MB dose) because of changes in FDA-regulated color balance that occur with higher fluence rate. Finally, opaque and semiopaque structures overlying vessels and the myocardium can lead to false-positive filling defects, making the Color-NIR Merge images of paramount importance for interpreting results.

The dual-channel capability of the imaging system would be particularly useful with acute intravascular thrombi. Thrombus formation is a key event in the pathogenesis of acute occlusion of the coronary arteries,${ }^{28}$ yet there is presently no method for its sensitive detection. The 700-nm MB NIR fluorescence did not interfere with the $800-\mathrm{nm}$ NIR fluorescence from platelets, permitting real-time and simultaneous assessment of thrombus size, arterial patency, and myocardial perfusion and thus the opportunity for intraoperative intervention. One can envision that with the development of additional targeted 800-nm fluorophores, such as those specific for cardiac stem cells, fibrosis, or other important features, cardiac surgeons could use the 800-nm channel to tailor image guidance during cardiac surgery.

One major limitation of the simple reflectance imaging system used in this study is depth penetration and surface weighting of fluorescence intensity. Although it can detect fluorescent lymph nodes through up to $1 \mathrm{~cm}$ of solid tissue $\mathrm{e}^{29}$ and $5 \mathrm{~cm}$ of lung tissue ${ }^{30}$ against a low autofluorescent background, it is unlikely that our imaging system will be able to detect subendocardial or septal ischemia in the absence of epicardial ischemia. Recent advances in optical tomography suggest that depth penetration up to $2 \mathrm{~cm}$ might someday be possible, although no study to date has attempted to apply tomography to moving objects, such as the heart.

Finally, the decay of MB NIR fluorescence from the myocardium is a complex function of physiologic, chemical, and optical variables. MB is sensitive to the redox state and in the presence of a reducing agent is converted to the colorless, nonfluorescent chemical leucomethylene blue. Leucomethylene blue is also uncharged, which permits diffusion out of myocytes and clearance from the body. Thus an added feature of $\mathrm{MB}$ imaging of the heart might be a direct visual assessment of the redox state, with a highly reducing environment leading to lower peak fluorescence and a more rapid return to baseline values and a highly oxidizing environment leading to high peak fluorescence and a slower return to baseline. Studies exploring whether these simple quantita- tive measurements might provide the cardiac surgeon with additional information about the oxidative (ie, ischemic) state of the heart are ongoing.

In summary, MB as a 700-nm NIR fluorophore for visualization of coronary arteriography and myocardial perfusion, independent 800-nm NIR fluorophores for visualization of other physiologic processes, and a dual-channel intraoperative NIR fluorescence imaging system provide unprecedented image guidance during cardiac surgery.

We thank Barbara L. Clough and Alice Gugelmann for editing and Eugenia Trabucchi for administrative assistance.

\section{References}

1. Chesebro JH, Clements IP, Fuster V, Elveback LR, Smith HC, Bardsley WT, et al. A platelet-inhibitor-drug trial in coronary-artery bypass operations: benefit of perioperative dipyridamole and aspirin therapy on early postoperative vein-graft patency. N Engl J Med. 1982;307:73-8.

2. FitzGibbon GM, Burton JR, Leach AJ. Coronary bypass graft fate: angiographic grading of 1400 consecutive grafts early after operation and of 1132 after one year. Circulation. 1978;57:1070-4.

3. Frangioni JV. In vivo near-infrared fluorescence imaging. Curr Opin Chem Biol. 2003;7:626-34.

4. Tanaka E, Choi HS, Fujii H, Bawendi MG, Frangioni JV. Image-guided oncologic surgery using invisible light: completed pre-clinical development for sentinel lymph node mapping. Ann Surg Oncol. 2006;13:1671-81.

5. Ohnishi S, Lomnes SJ, Laurence RG, Gogbashian A, Mariani G, Frangioni JV. Organic alternatives to quantum dots for intraoperative near-infrared fluorescent sentinel lymph node mapping. Mol Imaging. 2005;4:172-81.

6. Soltesz EG, Laurence RG, De Grand AM, Cohn LH, Mihaljevic T, Frangioni JV. Image-guided quantification of cardioplegia delivery during cardiac surgery. Heart Surg Forum. 2007;10:E381-6.

7. Tanaka E, Ohnishi S, Laurence RG, Choi HS, Humblet V, Frangioni JV. Realtime intraoperative ureteral guidance using invisible near-infrared fluorescence. J Urol. 2007; 178:2197-202.

8. Tanaka E, Choi HS, Humblet V, Ohnishi S, Laurence RG, Frangioni JV. Realtime intraoperative assessment of the extrahepatic bile ducts in rats and pigs using invisible near-infrared fluorescent light. Surgery. 2008;144:39-48.

9. Flaumenhaft R, Tanaka E, Graham GJ, De Grand AM, Laurence RG, Hoshino K, et al. Localization and quantification of platelet-rich thrombi in large blood vessels with near-infrared fluorescence imaging. Circulation. 2007;115: 84-93.

10. Desai ND, Miwa S, Kodama D, Koyama T, Cohen G, Pelletier MP, et al. A randomized comparison of intraoperative indocyanine green angiography and transittime flow measurement to detect technical errors in coronary bypass grafts. $J$ Thorac Cardiovasc Surg. 2006;132:585-94.

11. Reuthebuch O, Haussler A, Genoni M, Tavakoli R, Odavic D, Kadner A, et al. Novadaq SPY: intraoperative quality assessment in off-pump coronary artery bypass grafting. Chest. 2004;125:418-24.

12. Taggart DP, Choudhary B, Anastasiadis K, Abu-Omar Y, Balacumaraswami L, Pigott DW. Preliminary experience with a novel intraoperative fluorescence imaging technique to evaluate the patency of bypass grafts in total arterial revascularization. Ann Thorac Surg. 2003;75:870-3.

13. De Grand AM, Frangioni JV. An operational near-infrared fluorescence imaging system prototype for large animal surgery. Technol Cancer Res Treat. 2003;2: 553-62.

14. Sens R, Drexhage KH. Fluorescence quantum yield of oxazine and carbazine laser dyes. J Luminesc. 1981;24:709-12.

15. Tsopelas C, Sutton R. Why certain dyes are useful for localizing the sentinel lymph node. J Nucl Med. 2002;43:1377-82.

16. Nakayama A, Bianco AC, Zhang CY, Lowell BB, Frangioni JV. Quantitation of brown adipose tissue perfusion in transgenic mice using near-infrared fluorescence imaging. Mol Imaging. 2003;2:37-49.

17. Fabricius AM, Gerber W, Hanke M, Garbade J, Autschbach R, Mohr FW. Early angiographic control of perioperative ischemia after coronary artery bypass grafting. Eur J Cardiothorac Surg. 2001;19:853-8. 
18. Khan NE, De Souza A, Mister R, Flather M, Clague J, Davies S, et al. A randomized comparison of off-pump and on-pump multivessel coronary-artery bypass surgery. N Engl J Med. 2004;350:21-8.

19. Falk V, Walther T, Kitzinger H, Rauch T, Diegeler A, Autschbach R, et al. An experimental approach to quantitative thermal coronary angiography. Thorac Cardiovasc Surg. 1998;46:25-7.

20. Louagie YA, Haxhe JP, Buche M, Schoevaerdts JC. Intraoperative electromagnetic flowmeter measurements in coronary artery bypass grafts. Ann Thorac Surg. 1994;57:357-64.

21. Matre K, Birkeland S, Hessevik I, Segadal L. Comparison of transit-time and Doppler ultrasound methods for measurement of flow in aortocoronary bypass grafts during cardiac surgery. Thorac Cardiovasc Surg. 1994;42:170-4.

22. Canver CC, Cooler SD, Murray EL, Nichols RD, Heisey DM. Clinical importance of measuring coronary graft flows in the revascularized heart. Ultrasonic or electromagnetic? J Cardiovasc Surg (Torino). 1997;38: 211-5.

23. D'Ancona G, Karamanoukian HL, Ricci M, Schmid S, Bergsland J, Salerno TA. Graft revision after transit time flow measurement in off-pump coronary artery bypass grafting. Eur J Cardiothorac Surg. 2000;17:287-93.
24. Hol PK, Fosse E, Mork BE, Lundblad R, Rein KA, Lingaas PS, et al. Graft control by transit time flow measurement and intraoperative angiography in coronary artery bypass surgery. Heart Surg Forum. 2001;4:254-8.

25. Detter C, Wipper S, Russ D, Iffland A, Burdorf L, Thein E, et al. Fluorescent cardiac imaging: a novel intraoperative method for quantitative assessment of myocardial perfusion during graded coronary artery stenosis. Circulation. 2007;116:1007-14.

26. Pinero A, Illana J, Garcia-Palenciano C, Canizarese F, Canteras M, Canadillas V, et al. Effect on oximetry of dyes used for sentinel lymph node biopsy. Arch Surg. 2004;139:1204-7.

27. Martindale SJ, Stedeford JC. Neurological sequelae following methylene blue injection for parathyroidectomy. Anaesthesia. 2003;58:1041-2.

28. Gawaz M. Role of platelets in coronary thrombosis and reperfusion of ischemic myocardium. Cardiovasc Res. 2004;61:498-511.

29. Kim S, Lim YT, Soltesz EG, De Grand AM, Lee J, Nakayama A, et al. Near-infrared fluorescent type II quantum dots for sentinel lymph node mapping. Nat Biotechnol. 2004;22:93-7.

30. Soltesz EG, Kim S, Laurence RG, DeGrand AM, Parungo CP, Dor DM, et al. Intraoperative sentinel lymph node mapping of the lung using near-infrared fluorescent quantum dots. Ann Thorac Surg. 2005;79:269-77. 